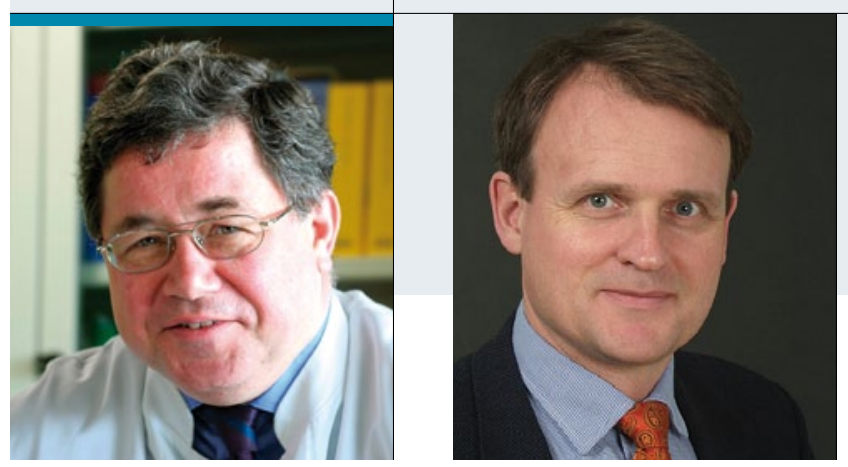

„Trotz offensichtlich vorhandener Mittel wird die

Unterversorgung von Allergikern nicht behoben

- im Gegenteil!"

Prof. Dr. Hans F. Merk, Hautklinik der Medizinischen Fakultät, Universitätsklinikum der RWTH Aachen Prof. Dr. Thilo Jakob, Allergieabteilung \& Forschergruppe Allergologie, Universitäts-Hautklinik Freiburg

\title{
Vorsicht: falsche Versprechungen!
}

n dem Brief einer Krankenkasse an ihre Mitglieder, den Sie - inklusive Kommentar von Prof. Dr. Ludger Klimek - auf S. 135 in diesem Heft nachlesen können, sind interessante Neuerungen zu erfahren, die uns entgangen sein müssen. Das Datum des Briefes ist übrigens nicht der 1. April. Die Kasse wendet sich an Mitglieder, die „wegen einer Allergie/ Asthma/Neurodermitis in ärztlicher Behandlung “ waren und auf eine „medikamentengestützte Therapie verzichten wollen“. Diesen Patienten wird nahegelegt, an dem Programm „Allergiefrei in 4 Wochen“ teilzunehmen. Diese Therapie beruhe ,auf einer Ernährungsweise, die den Säure-Base-Haushalt des Körpers wieder ins Gleichgewicht bringt“. „Die bisherigen Erfahrungen mit dem neuen Programm zeigen, dass bei Allergien gleich welcher Art ... die Beschwerden dauerhaft gelindert werden können. "Die Gesamtkosten belaufen sich auf $560 €$, an denen sich die Kasse mit $360 €$ beteiligen will. Dieses Angebot demonstriert, zu welch merkwürdigen Aktivitäten der gegenwärtig bestehende Überschuss im Gesundheitsfond führt. Trotz offensichtlich vorhandener Mittel wird die Unterversorgung von Allergikern nicht behoben - im Gegenteil. Diesen Patienten werden Alternativen angeboten, die aufgrund falscher Versprechungen sogar gefährdend sein können!

Auf dem Euregio-Allergie-Symposium, das kürzlich in Köln stattfand, konnte Dr. Carl Schönherr auf eine interessante Entwicklung in der KV Nordrhein aufmerksam machen: Diese war und ist bereit, ein qualifikationsgebundenes Zusatzvolumen (QZV) für Allergologie und Hyposensibilisierung einzurichten. Auf die kompetenten Arztgruppen aufgeteilt, ergaben sich als Honorare für das QZV Allergologie: 27,94 $€$ bei Hausärzten, $38,92 €$ bei Kinderärzten, $26,67 €$ bei Pneumologen, 31,51€ bei Dermatologen und 27,08 € bei HNO-Ärzten und für das QZV Hyposensibilisierung: $26,70 €$ bei Hausärzten, $24,94 €$ bei Kinderärzten, $18,77 €$ bei Pneumologen, 19,70 € bei Dermatologen und $19,53 €$ bei HNO-Ärzten. Die Regelung hätte zu einer Abstaffelung des Regelleistungsvolumen geführt (Hausärzte: 0,04€[<-1\%], Kinderärzte: 0,24€ [-1\%], HNO-Ärzte: $1,09 €[-5 \%]$, Dermatologen: $1,57 €[-13 \%$,
Pneumologen: 3,02€ [-9\%]). Dieser Regelung haben nur die Vertretungen der Hausärzte und Kinderärzte zugestimmt, was nun zur Folge hat, dass ein QZV für Allergologie und Hyposensibilisierung nur für entsprechend qualifizierte Hausärzte (Allergologie: 40,03 $€$, SIT: $28,34 €$ ) und Kinderärzte (Allergologie: 40,15€, SIT: 27,54€) eingeführt wurde. Besonders begrüßenswert ist, dass die KV Nordrhein aufgrund der Erkenntnisse der von Prof. Dr. Jürgen Wasem auf Anregung des AeDA durchgeführten Studie zur Versorgung allergologischer Patienten den offenkundigen Mehrbedarf bei den anstehenden Verhandlungen mit den Kassen ins Gespräch bringen will. In Zukunft sollte aber eine bessere Abstimmung zwischen Berufsverbänden und ihren jeweiligen wissenschaftlichen Gesellschaften bestehen, die - wie z. B. die Deutsche Dermatologische Gesellschaft - immer wieder ihr besonderes Interesse an der Stützung allergologischer Tätigkeiten zugesichert haben.

In der Arbeitsgruppe Allergocologne wurde kürzlich die In-vitro-Diagnostik durch Allergologen diskutiert und ihre bedeutende Stellung in Diagnostik und Monitoring begründet. Daraus ist das Positionspapier „Allergologie 2013“ entstanden (S. 128). Pünktlich zu dem in diesem Jahr etwas verspäteten Start des Pollenflugs finden Sie auf S. 112 einen Artikel über die Erfahrungen mit dem „elektronischen Pollentagebuch“.

Das Thema HNO und Allergie lässt uns auch an den in diesem Heft gewürdigten runden Geburtstag von Prof. Dr. Wolfgang Schlenter denken (S. 138), der sich für die Allergologie nicht nur durch seine medizinischen und technischen Beiträge zur Entwicklung der nasalen Provokationstechnik verdient gemacht hat, sondern auch seit vielen Jahren die Funktion des AeDA-Schatzmeisters ausübt und im vergangenen Jahr mit der Viktor-Ruppert-Medaille ausgezeichnet wurde. Die Herausgeber schließen sich der Gratulation an und wünschen den Lesern viel Interesse beim Lesen des Allergo Journal.
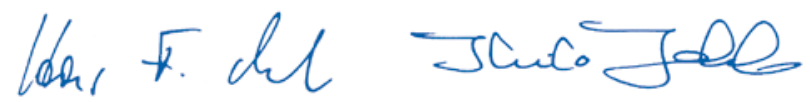